\title{
OPTIMIZATION OF DECOMPRESSIVE CRANIECTOMY BASED ON FINITE ELEMENT SIMULATIONS
}

\author{
Mate Hazay ${ }^{a, *}$, Bernadett Bakos ${ }^{a}$, Peter Jozsef Toth $^{b}$, Andras Buki ${ }^{b}$, \\ $\operatorname{IMRE~BOJTAR}^{a}$ \\ ${ }^{a}$ Department of Structural Mechanics, Budapest University of Technology and Economics, Muegyetem rkp. 3, \\ Budapest, Hungary \\ ${ }^{b}$ Department of Neurosurgery, University of Pecs, Ret str. 2, Pecs, Hungary \\ * corresponding author: hazay.mate@epito.bme.hu
}

Abstract. Decompressive Craniectomy (DC) is a neurosurgical procedure which is often applied to decrease the intracranial pressure (ICP), even if its optimal execution in terms of the size and location of the skull opening is not known. The current research focuses on DC from a biomechanical perspective. A finite element (FE) modelling strategy is applied where patient-specific head models are developed. These numerical models are used to perform virtual experiments where DC is simulated several times with skull openings having different size and location. During the simulations ICP, stress and strain distributions in the brain tissue are monitored in the function of the skull opening details. In the knowledge of these objective functions suggestions could be made regarding the applied optimization procedure which can lead to the identification of optimal DC scenarios in the future.

KEYworDS: intracranial pressure, numerical simulations, patient-specific head models, decompressive craniectomy.

\section{INTRODUCTION}

Traumatic brain injuries (TBIs) have a devastating global epidemiological importance [1. After a TBI the swelling of the brain tissue may occur which can lead to the increment of the intracranial pressure (ICP) [2] Increased ICP can cause secondary injury which can lead to serious neurological damages and death, therefore an important goal of the treatment procedure is the reduction of the pressure 2. In order to decrease $\mathrm{ICP}$, the so-called decompressive craniectomy (DC) neurosurgical operation can be performed, where a portion of the skull is removed and the underlying dura mater is opened to allow a bulging deformation of the parenchyma [3]. Due to the expansion of the brain tissue, the ICP decreases, however, axons may be subjected to extreme stretching which can be responsible for the unfavourable outcome which was observed after patients had been treated with DC [4]. Currently, the effective application of DC is challenging since its optimal execution in terms of the size and the location of the skull opening is controversial [5].

Our current research focuses on DC from a biomechanical perspective. Our main goal is to analyse the biomechanical behaviour of the soft and highly deformable brain tissue in the function of the size and location of the skull opening. In the knowledge of the pressure reduction and strain distributions suggestions can be made about the optimization procedure which could be applied in the future in order to identify optimal DC scenarios, where significant pressure reduction could be accomplished without the excessive stretches of the brain tissue.

\section{Methods}

In order to analyse the mechanical response of the brain, a finite element (FE) modelling strategy is applied which includes the development of head models with patient-specific geometry. Its preparation starts with nonlinear medical image registration where the CT scans of a healthy volunteer are morphed to the preoperative CT scans of the patient based on the patient's cranial shape [6] using B-splines [7] in 3D Slicer environment 8 . In the resulting $\mathrm{CT}$ scans the cranial shape approximately agrees with the patient's cranial shape, but the shape of the intracranial anatomic parts represent an average healthy condition [9]. On these scans the different anatomic parts are distinguished by segmentation performed in 3D Slicer using threshold based semi-automatic method. After the pixelwise segmentation, the obtained surface meshes are smoothed and corrected by VCG reconstruction [10] in Meshlab 11. Smoothed surface models are converted to 3D CAD geometry in ANSYS ${ }^{\circledR}$ SpaceClaim (Release 18.0, ANSYS, Inc., PA, USA) and the obtained geometry is imported to ANSYS ${ }^{\circledR}$ Workbench (Release 18.0, ANSYS, Inc., PA, USA) where the finite element models are developed. Similarly to previous research [12, the geometry includes the skull, dura mater, brain tissue, outer cerebrospinal fluid (CSF) space, lateral ventricles and the falx cerebri. However, the outer CSF space and the ventricles are represented as gaps in the geometry, while the supporting effect of the CSF on the surface of the parenchyma is taken into account by (i) a pressure load with $5 \mathrm{mmHg}$ intensity (approximating an initial healthy state) and (ii) an 


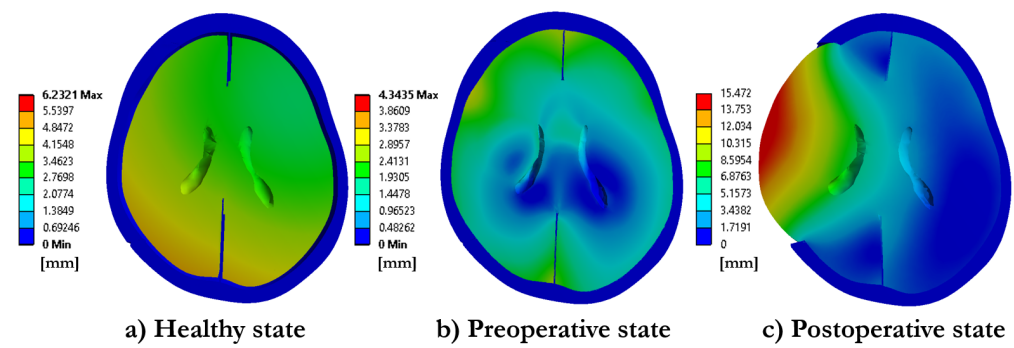

Figure 1. Obtained displacements at the main steps of the FE analysis.

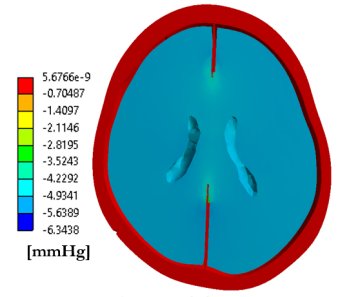

a) Healthy state

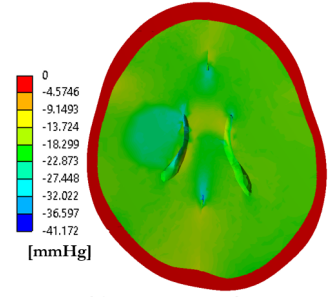

b) Preoperative state

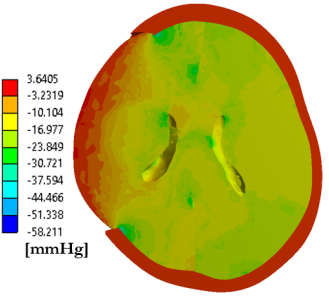

c) Postoperative state

Figure 2. Obtained ICP at the main steps of the FE analysis.

elastic spring support (modelling the increased pressure at the surfaces due to swelling). In the model the brain tissue is considered as a homogeneous, isotropic domain having a hyperelastic $2^{\text {nd }}$-order Ogden material model which was applied in previous research [13, while the skull and dura is considered as a linear elastic continuum 9 .

In order to separate FE mesh generation from the unimportant geometric features, a virtual topology is constructed in ANSYS by generating virtual cells. In case of this highly irregular geometry, the FE mesh is created by the so-called patch independent algorithm of the ANSYS ${ }^{\circledR}$ Workbench. As a result, a FE mesh is obtained with good quality tetrahedron elements for the skull and brain tissue and triangular elements for the falx cerebri. Accordingly, the total number of elements in the models is between 100000-120000.

During the analysis each node of the skull is constrained against translational displacements, while the brain tissue is supported by the above-mentioned weak springs representing the effect of CSF, and a frictionless contact is established between the brain and skull and between the brain and falx.

Displacement and ICP distributions obtained at the main steps of the FE analysis are illustrated in Fig. 1 and Fig. 2. The simulations start from an initial (unloaded) reference state, then the boundaries of the brain tissue are subjected to the above-mentioned $5 \mathrm{mmHg}$ pressure in order to reach an approximate healthy state (Fig. 1.a, Fig. 2 a). Afterwards, the brain swelling due to edema is simulated by an artificial thermal expansion in order to reach the preoperative (i.e. injured) state (Fig. 1 b, Fig. 2 b). Finally, the skull opening is performed by prescribed displacements of the bone flap and the corresponding bulging displacement is reached at the postoperative state (Fig. 1]c, Fig. 2 c). In order to simulate that certain magnitude of CSF flows out during the operation, near the craniectomy the $5 \mathrm{mmHg}$ initial pressure and the spring elements are killed at the time of the skull opening.

These models are calibrated and validated against clinical measurements by reconstructing real-life DC cases where the observed (i.e. measured at the clinic) and predicted (i.e. obtained by FE simulations) ICP and deformations are compared on a qualitative and quantitative manner [9]. During the calibration the magnitude of the fictive thermal loading is determined based on the equality of the observed and predicted ICPs near the lateral ventricles at the preoperative state. Results of the validation procedure are detailed elsewhere [9].

In this work two different models (one with unilateral and the other one with bifrontal skull opening) were applied to perform virtual experiments where DC is simulated several times with different sized skull openings. Following this procedure, it is monitored how the biomechanical behaviour of the brain tissue depends on the size and location of the skull opening.

\section{Results}

Some fundamental aspects regarding the biomechanical behaviour of the brain are shown in Fig. 3 in terms of displacements (left), shear strains (middle) and principal strain (right). It can be seen that even for a relatively low $(18 \mathrm{mmHg})$ preoperative pressure the brain tissue suffers relatively large bulging deformations (Fig. 3 a). Due to these excessive deformations the soft tissue is subjected to two different loading phenomena. Firstly, a relatively large shear zone occurs (i) along the perimeter of the skull opening and (ii) between the ventricles and the edges of the skull opening (Fig. 3.b). Moreover, a relatively large volume of the brain tissue is under tension (Fig. 3 c), although 


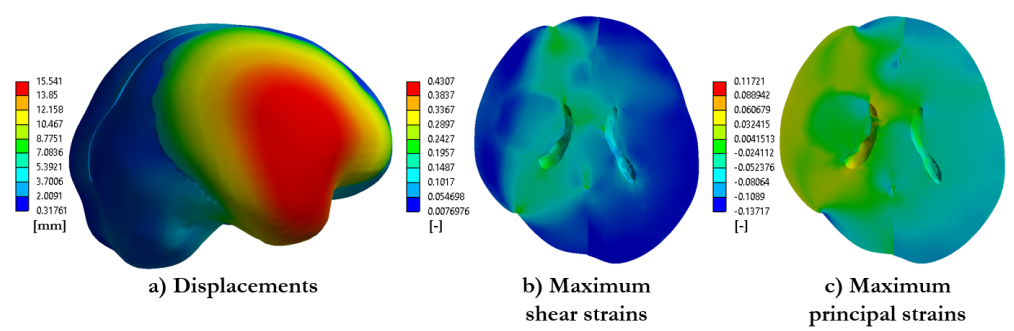

FiguRE 3. Biomechanical behavior of the brain tissue during DC.

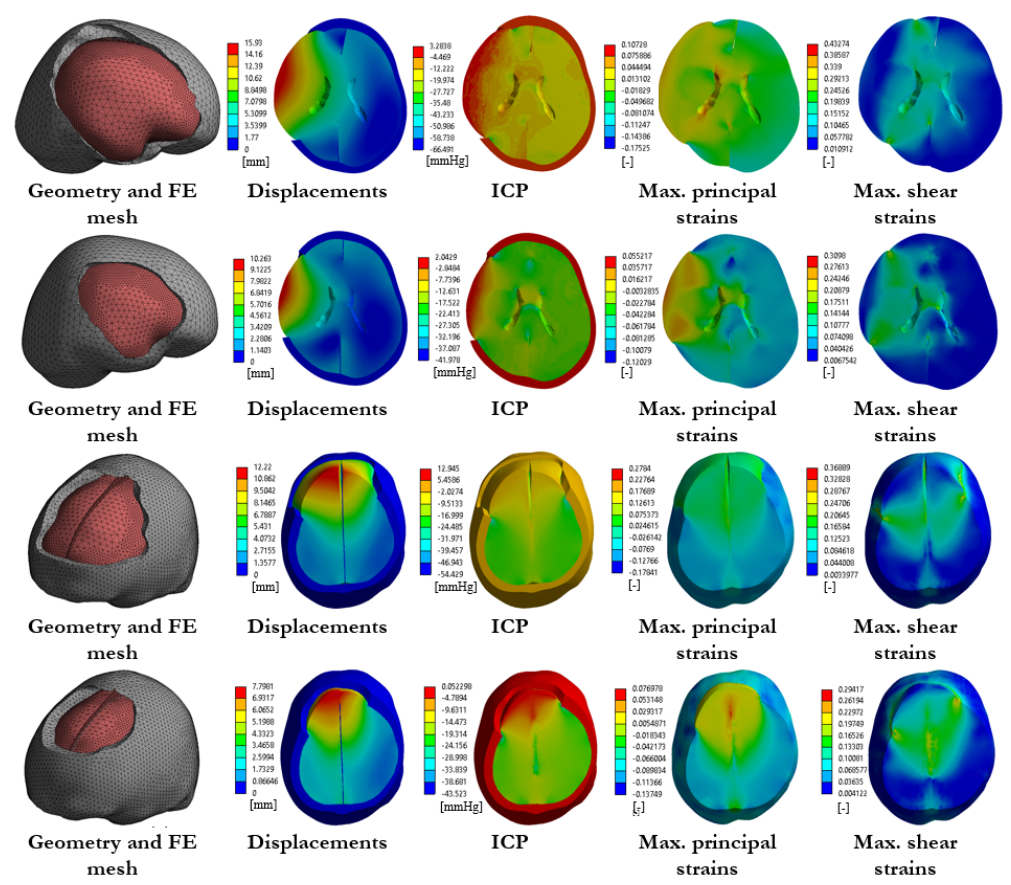

Figure 4. Geometry and FE results in case of different DC scenarios.

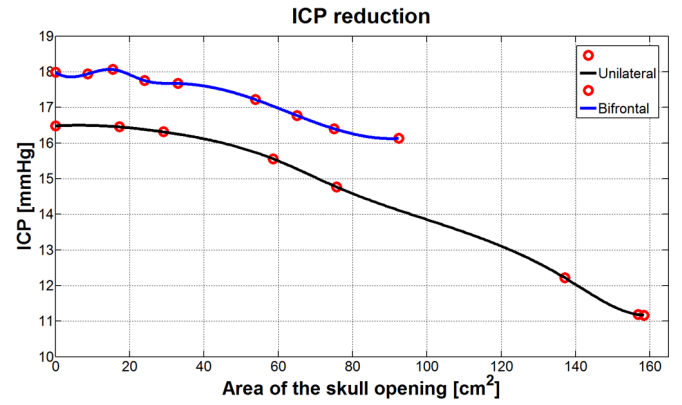

FiguRE 5. ICP reduction in the function of skull opening area.
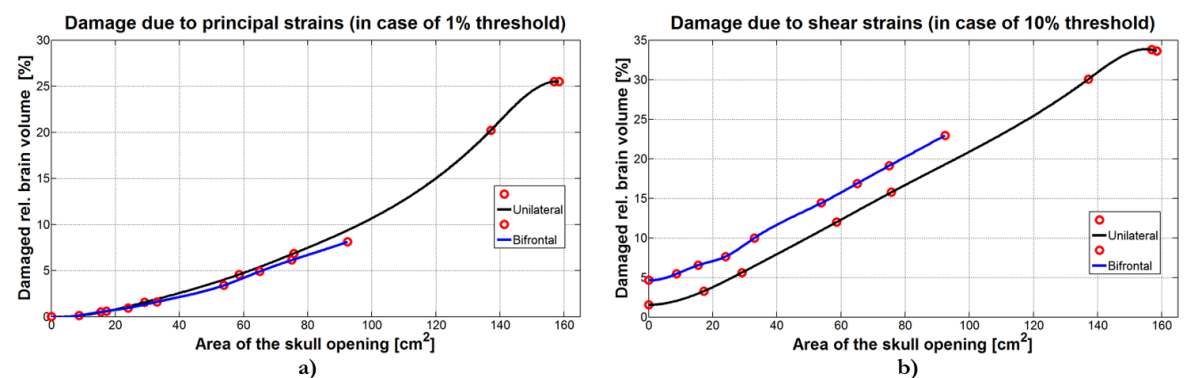

Figure 6. Tendencies of damaged brain volume in case of different skull opening area. 
it is expected that even minimal tensile strains can cause neurological damage if they maintained for a long duration such as in the postoperative stage [6]. Based on these observations, besides ICP and total displacements, the shear strains and the maximum principal strains were continuously monitored during the simulation of different DC scenarios. Regarding these parameters, some illustrative results obtained by FE simulations in case of different skull openings are shown in Fig. 4. Based on the results of virtual experiments the pressure reduction seems to have an approximate linear dependence on the skull opening area as it is shown in Fig. 5.

Tendencies of the damaged relative brain volume in case of assumed strain thresholds are shown in Fig. 6 due to maximum principal strains (Fig. 6.a) and shear strains (Fig. 6 b).

In Fig. 5 and Fig. 6 multiple objective functions can be seen which should be minimized. Since these are monotonic competitive functions the so-called Paretofront (i.e. the set of solutions which are optimal in a sense that one objective function (e.g. in Fig. 6) can take a lower value if and only if the other objective function (in Fig. 5 takes a larger value) consists of infinitely many points.

\section{Discussion}

In this work numerical simulations of decompressive craniectomy were performed with different sized unilateral and bifrontal skull openings, in order to analyse the mechanical behaviour of the brain tissue in the function of the skull opening details. It was observed that the pressure reduction depends on the area of the skull opening in an approximately linear way (Fig. 5). However, as the area of the skull opening increases the damaged brain volume due to maximum principal and maximum shear strains increases as well (Fig. 6). These multiple monotonic objective functions lead to infinitely many possible optimal solutions with the Pareto-type optimization. Therefore, it is suggested to define a target ICP value as a constraint and determine the optimal size of the skull opening based on the skull opening area versus ICP objective function (Fig. 5). In this way it could be avoided to apply a larger skull opening than the necessary which would cause unnecessarily large deformations and damage of the brain tissue.

\section{ACKNOWLEDGEMENTS}

This research was supported by the UNKP-17-3-1 New Excellence Program and the BME-Biotechnology FIKP grant (BME FIKP-BIO) of the Ministry of Human Capacities.

\section{REFERENCES}

[1] T. Frieden, D. Houry, G. Baldwin. Report to congress on traumatic brain injury in the united states: Epidemiology and rehabilitations, 2010.

[2] S. Badri, J. Chen, J. Barber, et al. Mortality and long-term functional outcome associated with intracranial pressure after traumatic brain injury. Intensive Care Medicine 38(11):1800-1809, 2012. DOI:10.1007/s00134-012-2655-4

[3] I. Timofeev, M. Czosnyka, J. Nortje, et al. Effect of decompressive craniectomy on intracranial pressure and cerebrospinal compensation following traumatic brain injury. Journal of Neurosurgery 108(1):66-73, 2008. DOI:10.3171/JNS/2008/108/01/0066

[4] D. Cooper, J. Rosenfeld, L. Murray, et al. Decompressive craniectomy in diffuse traumatic brain injury. New England Journal of Medicine 364(16):14931502, 2011. DOI:10.1056/NEJMoa1102077

[5] A. Kolias, P. Kirkpatrick, P. Hutchinson. Decompressive craniectomy: Past, present and future. New England Journal of Medicine 364(16):1493-1502, 2011. DOI:10.1038/nrneurol.2013.106.

[6] H. von Holst, X. Li, S. Kleiven. Increased strain levels and water content in brain tissue after decompressive craniotomy. Acta Neurochirurgica 154(9):1583-1593, 2012. DOI:10.1007/s00701-012-1393-2

[7] S. Gu, X. Meng, F. Sciurba, et al. Bidirectional elastic image registration using b-spline affine transformation. Computerized Medical Imaging and Graphics 38(4):306314, 2014. DOI:10.1016/j.compmedimag.2014.01.002

[8] S. Pieper, M. Halle, R. Kikinis. 3d slicer. Proceedings of the 2nd IEEE International Symposium on Biomedical Imaging: Nano to Macro, 2004 Apr 15-18, Arlington, VA, USA 1:632-635, 2004.

[9] M. Hazay, A. Varga, E. Nagy, et al. Finite element reconstruction of decompressive craniectomy. Biomechanica Hungarica -(-):in press, 2018.

[10] M. Nabil, M. Betro, M. Metwally. 3d reconstruction of ancient egyptian rock-cut tombs: the case of m.i.d.a.n.05. In the Proceedings of the XXIV International CIPA Symposium pp. 443-447, 2013. 2013 Sep 2-6, Strasbourg, France.

[11] P. Cignoni, M. Callieri, M. Corsini, et al. Meshlab: an open-source mesh processing tool. In the Proceedings of the 6th Eurographics Italian Chapter Conference pp. 129-136, 2008. 2018 Jul 2-4, Salermo, Italy.

[12] X. Li, H. von Holst. Finite element modeling of decompressive craniectomy (dc) and its clinical validation. Advances in Biomedical Science and Engineering 2(1):1-9, 2015. DOI:10.15764/ABSE.2015.01001

[13] T. Fletcher, B. Wirthl, A. Kolias, et al. Modelling of brain deformation after decompressive craniectomy. Annals of Biomedical Engineering 44(12):3495-3509, 2016. DOI:10.1007/s10439-016-1666-7 